\title{
Maternal effects shape the alternative splicing of parental alleles in reciprocal cross hybrids of Megalobrama amblycephala $\times$ Culter alburnus
}

Li Ren ${ }^{1,2+}$, Xiaojing Yan ${ }^{1,2+}$, Xin Gao ${ }^{1,2}$, Jialin Cui ${ }^{1,2}$, Pengcheng Yan ${ }^{3}$, Chang Wu ${ }^{1,2}$, Wuhui Li $i^{1,2}$ and Shaojun Liu ${ }^{1,2^{*}}$

\begin{abstract}
Background: Maternal effects contribute to adaptive significance for shaping various phenotypes of many traits. Potential implications of maternal effects are the cause of expression diversity, but these effects on mRNA expression and alternative splicing (AS) have not been fully elucidated in hybrid animals.

Results: Two reciprocal cross hybrids following hybridization of Megalobrama amblycephala (blunt snout bream, BSB) and Culter alburnus (topmouth culter, TC) were used as a model to investigate maternal effects. By comparing the expression of BSB- and TC- homoeologous genes between the two reciprocal cross hybrids, we identified 49348 differentially expressed BSB-homoeologous genes and 54-354 differentially expressed TC-homoeologous genes. 2402, 2959, and 3418 AS events between the two reciprocal cross hybrids were detected in Illumina data of muscle, liver, and gonad, respectively. Moreover, 21,577 (TC-homoeologs) and 30,007 (BSB-homoeologs) AS events were found in the 20,131 homoeologous gene pairs of $\mathrm{TBF}_{3}$ based on PacBio data, while 30,561 (TC-homoeologs) and 30,305 (BSB-homoeologs) AS events were found in $\mathrm{BTF}_{3}$. These results further improve AS prediction at the homoeolog level. The various AS patterns in bmprza belonging to the bone morphogenetic protein family were selected as AS models to investigate the expression diversity and its potential effects to body shape traits.

Conclusions: The distribution of differentially expressed genes and AS in BSB- and TC-subgenomes exhibited various changes between the two reciprocal cross hybrids, suggesting that maternal effects were the cause of expression diversity. These findings provide a novel insight into mRNA expression changes and AS under maternal effects in lower vertebrates.
\end{abstract}

Keywords: Maternal effects, Alternative splicing, Reciprocal cross hybridization, Differential expression, Homoeologous expression

\footnotetext{
* Correspondence: Isj@hunnu.edu.cn

${ }^{\dagger}$ Li Ren and Xiaojing Yan contributed equally to this work.

'State Key Laboratory of Developmental Biology of Freshwater Fish, Hunan

Normal University, Changsha 410081, Hunan, P.R. China

${ }^{2}$ College of Life Sciences, Hunan Normal University, Changsha 410081,

Hunan, P.R. China

Full list of author information is available at the end of the article
}

(c) The Author(s). 2020 Open Access This article is licensed under a Creative Commons Attribution 4.0 International License, which permits use, sharing, adaptation, distribution and reproduction in any medium or format, as long as you give appropriate credit to the original author(s) and the source, provide a link to the Creative Commons licence, and indicate if changes were made. The images or other third party material in this article are included in the article's Creative Commons licence, unless indicated otherwise in a credit line to the material. If material is not included in the article's Creative Commons licence and your intended use is not permitted by statutory regulation or exceeds the permitted use, you will need to obtain permission directly from the copyright holder. To view a copy of this licence, visit http://creativecommons.org/licenses/by/4.0/ The Creative Commons Public Domain Dedication waiver (http://creativecommons.org/publicdomain/zero/1.0/) applies to the data made available in this article, unless otherwise stated in a credit line to the data. 


\section{Background}

Maternal effects are the causal influence of the maternal genotype or phenotype on the phenotype of the offspring [1]. The maternal influence is generally in the form of maternal messenger RNAs that are partly made by maternal mitochondrial genes and shape the traits of hybrids including growth and starvation resistance, similar to that of maternal parents $[2,3]$. The definition of maternal effects is often extended to incorporate a diversity of other related phenomena (e.g. kin effects, genomic imprinting, uniparental extra-chromosomal inheritance) [1]. Some studies reported that the maternal effects associated with methyltransferase led to maternal genomic imprinting [4, 5], which referred to the phenomenon where individuals expressed only one copy of the maternal or paternal allele. More generally, it refers to parent-of-origin-dependent gene expression or effects [6,7]. Although biologists have known about the importance of these effects for decades, their influences on expression diversity in offspring have not been fully elucidated.

Alternative splicing (AS), including skipped exons (SE), retained introns (RI), alternative $5^{\prime}$ splice sites (A5SS), alternative 3' splice sites (A3SS), and alternative position (AP), generates multiple transcripts from the same gene by combining different exons. It expands transcriptome plasticity and proteomic diversity, thereby regulating gene expression at the post-transcriptional level [8]. Pre-mRNA splicing is largely co-transcriptional, and the alternative splice site choice is influenced by the RNA polymerase II elongation rate, chromatin remodelers, and histone deacetylase inhibitors $[9,10]$. Recent studies using Illumina and PacBio sequencing indicated that about 25,60 , and $90 \%$ of multiexon genes in Caenorhabditis elegans, Drosophila melanogaster, and human, respectively, undergo AS [11-13]. Changes in AS represent one of the major driving forces underlying the evolution of phenotypic differences across different species [14, 15]. However, few studies have focused on hybrids because of their complex regulation patterns [16].

Homoeologs are the orthologous gene pairs from two or more inbred hybrid parents in allodiploids and allopolyploids. The unequal expression of two or more homoeologs (also described as homoeolog expression bias), and the total expression level of a homoeolog pair similar to one of the two diploid parents (also described as expression level dominance), are contributed to the formation of various phenotypes, including heterosis [17-19]. Studies on homoeologs provide us a insight into the potential regulation mechanisms of various phenotypes. However, unlike in plants, intergeneric allodiploids are rarely found in vertebrates because of reproductive isolation and chromosomal pairing disorder during gamete formation, or hybrid individuals with failed to have offspring. However, two intergeneric reciprocal cross hybrids were previously obtained by the hybridization of Megalobrama amblycephala (BSB) and Culter alburnus (TC) [20, 21], which exhibited slight differences in body shape. In the present study, we detected differences in global expression (in both of two alleles) and homoeologous expression (in each of two alleles) between two reciprocal cross hybrids. We also predicted AS differences between the two homoeologs based on Illumina and PacBio data. Our results provide a comprehensive study of regulatory divergence under maternal effects.

\section{Results}

\section{Origin of reciprocal cross hybrids}

We first characterized the divergence of AS between two reciprocal cross hybrids $\left(\mathrm{BTF}_{3}\right.$ and $\left.\mathrm{TBF}_{3}\right)$, which were obtained from the self-crossing of respective reciprocal cross hybrids of $M$. amblycephala $(2 \mathrm{n}=48) \times C$. alburnus $(2 \mathrm{n}=48)$ [20, 21]. The genotype of chimeric offspring was determined as the allodiploid $(2 \mathrm{n}=48)$ with a 1:1 subgenome ratio with chromosome number and $45 \mathrm{~S}$ rDNA characteristics [20], in which the two types of $45 \mathrm{~S}$ rRNA were detected and belonged to species-specific sequences of $M$. amblycephala and C. alburnus, respectively [21] (Additional file 1: Table S1). The expression of mitochondrial genes in the two reciprocal cross hybrids was considered to be identical to that of the respective inbred female parents based on the mapped reads of transcriptome (Additional file 2: Table S2).

\section{Characteristic differences of the two subgenomes}

For the two inbred parental genomes (1.09 Gb in BSB and $1.02 \mathrm{~Gb}$ in $\mathrm{TC}$ ), the distributions of exon numbers and CDS lengths were obtained from 20,131 orthologous gene pairs (Additional file 3: Fig. S1). The average exon number in each gene was 8.83 in BSB and 9.64 in TC, while the average CDS length was $1525 \mathrm{bp}$ in BSB and $1654 \mathrm{bp}$ in TC. Focusing on same characteristic in the two parental genomes, the same exon number was found in 11,414 genes, and the same CDS length was detected in 6832 genes. Analysis of these results showed significant differences in exon number $(p<0.001)$ and CDS length $(p<0.001)$. Furthermore, strong associations with exon number and CDS length were detected in BSB $(r=$ $0.7435)$ and TC $(r=0.7768)$ (all $p<0.0001$ for Pearson correlation coefficients).

\section{Obtaining of long length transcripts and gene ontology analysis}

PacBio sequencing was used to detect AS events in reciprocal cross hybrids. A total of $21.22 \mathrm{~Gb}$ and $15.49 \mathrm{~Gb}$ data were obtained from $\mathrm{TBF}_{3}$ and $\mathrm{BTF}_{3}$, respectively, and an average of 12 and 13 CCSs and $3080 \mathrm{bp}$ and 
Table 1 Summary of full-length transcriptome data

\begin{tabular}{lll}
\hline & $\mathrm{TBF}_{3}$ & $\mathrm{BTF}_{3}$ \\
\hline The sequencing data (Gb) & 21.22 & 15.49 \\
Insert reads (Gb) & 2.02 & 1.30 \\
Average length of insert read (bp) & 3080.18 & 2936.23 \\
Average CCSs of insert reads & 12 & 13 \\
Number of consensus reads & 663,834 & 479,667 \\
Number of five prime reads & $622,119(93.72 \%)$ & $459,029(95.70 \%)$ \\
Number of three prime reads & $628,065(94.61 \%)$ & $456,107(95.09 \%)$ \\
Number of full-length reads & $586,075(88.29 \%)$ & $431,999(90.06 \%)$ \\
\hline
\end{tabular}

2936 bp average insert read lengths were detected in $\mathrm{TBF}_{3}$ and $\mathrm{BTF}_{3}$, respectively (Table 1 ). To detect the integrity of sequencing data, 663,834 $\mathrm{TBF}_{3}$ and 479,667 $\mathrm{BTF}_{3} 3^{\prime}$ and $5^{\prime}$ - untranslated regions were analyzed to determine whether the transcripts were full-length. Then, 586,075 (88.29\%) and 431,999 (90.06\%) full-length reads were detected in $\mathrm{TBF}_{3}$ and $\mathrm{BTF}_{3}$ (Table 1). After deleting redundant sequences, a total of 316,533 and 268,986 consensus reads were found in $\mathrm{TBF}_{3}$ and $\mathrm{BTF}_{3}$, respectively.

After mapping to the combined genome of the two inbred parents, the 314,298 consensus reads and 76,518 isoforms were obtained from the mapped results of $\mathrm{TBF}_{3}$, while 267,949 consensus reads with 82,083 isoforms were found in $\mathrm{BTF}_{3}$. An average 99.29 and $99.61 \%$ of mapping ratios were detected in $\mathrm{TBF}_{3}$ and $\mathrm{BTF}_{3}$, respectively. Then, the sequences of 11,026 genes in $\mathrm{TBF}_{3}$ and 11,448 genes in $\mathrm{BTF}_{3}$ were annotated with KEGG and GO databases (Additional file 4: Fig. S2). The 6071 genes shared between $\mathrm{TBF}_{3}$ and $\mathrm{BTF}_{3}$ were then focused on to help detect differences between the two.

\section{AS between two homoeologs}

To better characterize the differences between $\mathrm{TBF}_{3}$ and $\mathrm{BTF}_{3}$, we focused on AS events in BSB- and TC- homoeologs of the two reciprocal cross hybrids. A custom Python script was used to identify 30,007 AS events, and
7029 genes were mapped to the BSB-subgenome detected in 20,131 homoeologous genes of $\mathrm{TBF}_{3}$, while 21, 577 AS events and 5286 genes were mapped to the TCsubgenome (Table 2). We also detected 30,305 AS events and mapped 7271 genes to the BSB-subgenome of $\mathrm{BTF}_{3}$, while 30,562 AS events related to 6481 genes were mapped to the TC-subgenome (Table 2). Although the sequencing was performed in a mixture of three tissues, these data suggested a slight BSB-homoeolog expression bias in $\mathrm{TBF}_{3}$ but not in $\mathrm{BTF}_{3}$. Most of the AS events that occurred in hybrids were RI in TChomoeologs of $\mathrm{TBF}_{3}(18.96 \%)$ and $\mathrm{BTF}_{3}(26.49 \%)$, and BSB-homoeologs of $\mathrm{BTF}_{3}(26.70 \%)$. However, most AS events in BSB-homoeologs of $\mathrm{TBF}_{3}$ were SE (20.28\%) (Table 2). Although some errors in gene annotation may have led to an increased prediction of RI and SE, these results suggest that there are clear differences not only between the two reciprocal cross hybrids, but also between BSB- and TC-homoeologous genes. Then, we compared the number of AS events between in each orthologous gene pairs of two subgenomes. Among these, 1290 genes of $\mathrm{TBF}_{3}$ and 2302 genes of $\mathrm{BTF}_{3}$ were shown to possess AS events in both homoeologs. In $\mathrm{TBF}_{3}, 4862$ AS events supported by 6416 isoforms were mapped to the BSB-subgenome, while 4650 AS events supported by 6674 isoforms were mapped to the TCsubgenome (Fig. 1). In addition, we detected the 8054 AS events shared in orthologous gene pairs of BSBsubgenome and TC-subgenome of $\mathrm{TBF}_{3}$, while the 11, 024 AS events were shared in ones of $\mathrm{BTF}_{3}$.

\section{Expression changes led by maternal effects}

Comparison between $\mathrm{BTF}_{3}$ and $\mathrm{TBF}_{3}$, we identified 49 differentially expressed genes (DEGs) in BSBhomoeologous genes of liver, 186 DEGs in muscle, and 348 DEGs in gonad; this compared with 54 DEGs in TC-homoeologous genes of liver, 204 in muscle, and 354 in gonad (Fig. 2, Additional file 5: Table S3). The largest number of DEGs was found in gonad (3.58\% in BSB-homoeologs and $3.64 \%$ in TC-homoeologs) and the

Table 2 Summary of AS in BSB- and TC- homoeologs from full-length transcriptome data

\begin{tabular}{|c|c|c|c|c|c|c|c|c|}
\hline \multirow[t]{2}{*}{ AS types } & \multicolumn{2}{|c|}{ TC-homoeologs in $\mathrm{TBF}_{3}$} & \multicolumn{2}{|c|}{ BSB-homoeologs in $\mathrm{TBF}_{3}$} & \multicolumn{2}{|c|}{ TC-homoeologs in $\mathrm{BTF}_{3}$} & \multicolumn{2}{|c|}{ BSB-homoeologs in $\mathrm{BTF}_{3}$} \\
\hline & NO. of events (\%) & $\overline{\text { NO. of gene }}$ & NO. of events (\%) & NO. of gene & NO. of events (\%) & NO. of gene & NO. of events (\%) & NO. of gene \\
\hline $\begin{array}{l}\text { Alternative 3' } \\
\text { splice Site }\end{array}$ & $1430(6.63 \%)$ & 988 & $2026(6.75 \%)$ & 1439 & $1714(5.61 \%)$ & 1177 & 1877 (6.19\%) & 1374 \\
\hline $\begin{array}{l}\text { Alternative } 5^{\prime} \\
\text { splice Site }\end{array}$ & $1603(7.43 \%)$ & 1140 & $2384(7.94 \%)$ & 1625 & $1912(6.26 \%)$ & 1271 & $2122(7.00 \%)$ & 1533 \\
\hline Alternative site & $2520(11.68 \%)$ & 1409 & $3300(11.00 \%)$ & 1640 & 4001 (13.09\%) & 1912 & $3538(11.67 \%)$ & 1899 \\
\hline Exon skipping & 3869 (17.93\%) & 2137 & $6086(20.28 \%)$ & 3379 & 3685 (12.06\%) & 2050 & $5101(16.83 \%)$ & 3005 \\
\hline Retained Introns & 4091 (18.96\%) & 823 & $5140(17.13 \%)$ & 1842 & 8096 (26.49\%) & 3107 & 8092 (26.70\%) & 3181 \\
\hline Other & 8064 (37.37\%) & 2001 & 11,071 (36.89\%) & 2745 & $11,153(36.49 \%)$ & 2491 & 9575 (31.60\%) & 2861 \\
\hline Total & 21,577 & 5286 & 30,007 & 7029 & 30,561 & 6481 & 30,305 & 7271 \\
\hline
\end{tabular}



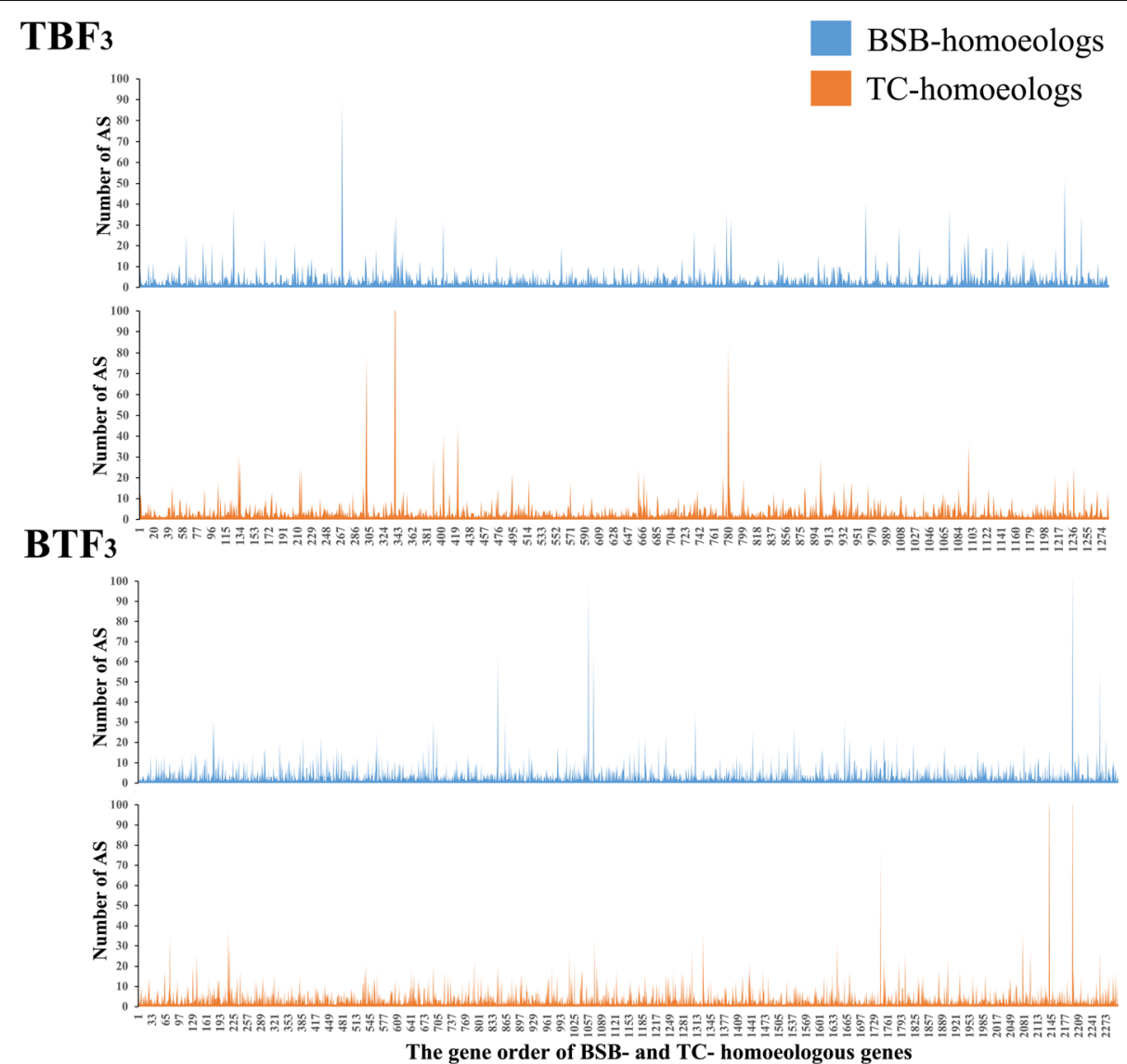

Fig. 1 Distribution of AS events observed in orthologous gene pairs of the two reciprocal cross hybrids. The difference on AS number was shown between BSB- and TC- homoeologs in each orthologous gene pairs

fewest were found in liver $(0.50 \%$ in BSB-homoeologs and $0.56 \%$ in TC-homoeologs) (Fig. 2, Additional file 5: Table S3).

We next focused on DEGs that were shared between BSB- and TC-homoeologs. The same up/down-regulated expression trends of the two homoeologs were exhibited among the three tissues (Fig. 2, Additional file 5: Table S3), indicating that similar differential expression trends occurred in both homoeologs. GO analysis showed that 90, 12, and 51 DEGs (the largest number in GO categories) were involved in the cellular process (GO: 0009987) (level 2) in gonad, liver, and muscle tissues, respectively (Additional file 6: Fig. S3 and S4). Some DEGs were also enriched in other functions, including metabolic process (GO: 0008152), response to stimulus (GO: 0050896), and biological regulation (GO: 0065007), while others were enriched in growth (GO: 0040007), immune system process (GO: 0002376), and reproduction (GO: 0000003) (Additional files 6 and 7: Fig. S3 and S4).

\section{Determination of AS in DEGs}

To further investigate the maternal effects on expression divergence, AS analysis was performed in homoeologous genes between the reciprocal cross hybrids. ASprofile detected 104 MXE and 3314 SE in gonad, 96 MXE and 2863 SE in liver, and 74 MXE and 2328 SE in muscle (Additional file 8: Table S4). Interestingly, 3103 (90.78\%) AS events were found in TC-homoeologous genes of gonad, while 315 (9.22\%) AS events were found in BSB-homoeologous genes. Moreover, 2706 (91.45\%) AS events were distributed in TC-homoeologous genes of liver, and the remaining 253 (8.55\%) AS events occurred in BSB-homoeologous genes. In muscle, 2205 (91.80\%) AS events occurred in TC-homoeologous genes compared with 197 (8.20\%) AS events in BSBhomoeologous genes (Additional file 8: Table S4). However, no RI, A5SS, or A3SS were identified in Illumina data. A total of 41,31, and 22 genes were detected as high AS events (number of AS types $\geq 5$ in each gene) from muscle, liver, and gonad, respectively. Among these, the five genes (ptprm, cast, exoc, myolb, and abila) with a high number of AS events were shared among the three tissues (Additional file 9: Fig. S5).

Combined analyses of AS and DEG, we identified AS events in 35 DEGs in gonad, 18 DEGs in muscle, and six DEGs in liver. Under different maternal effects, changes 


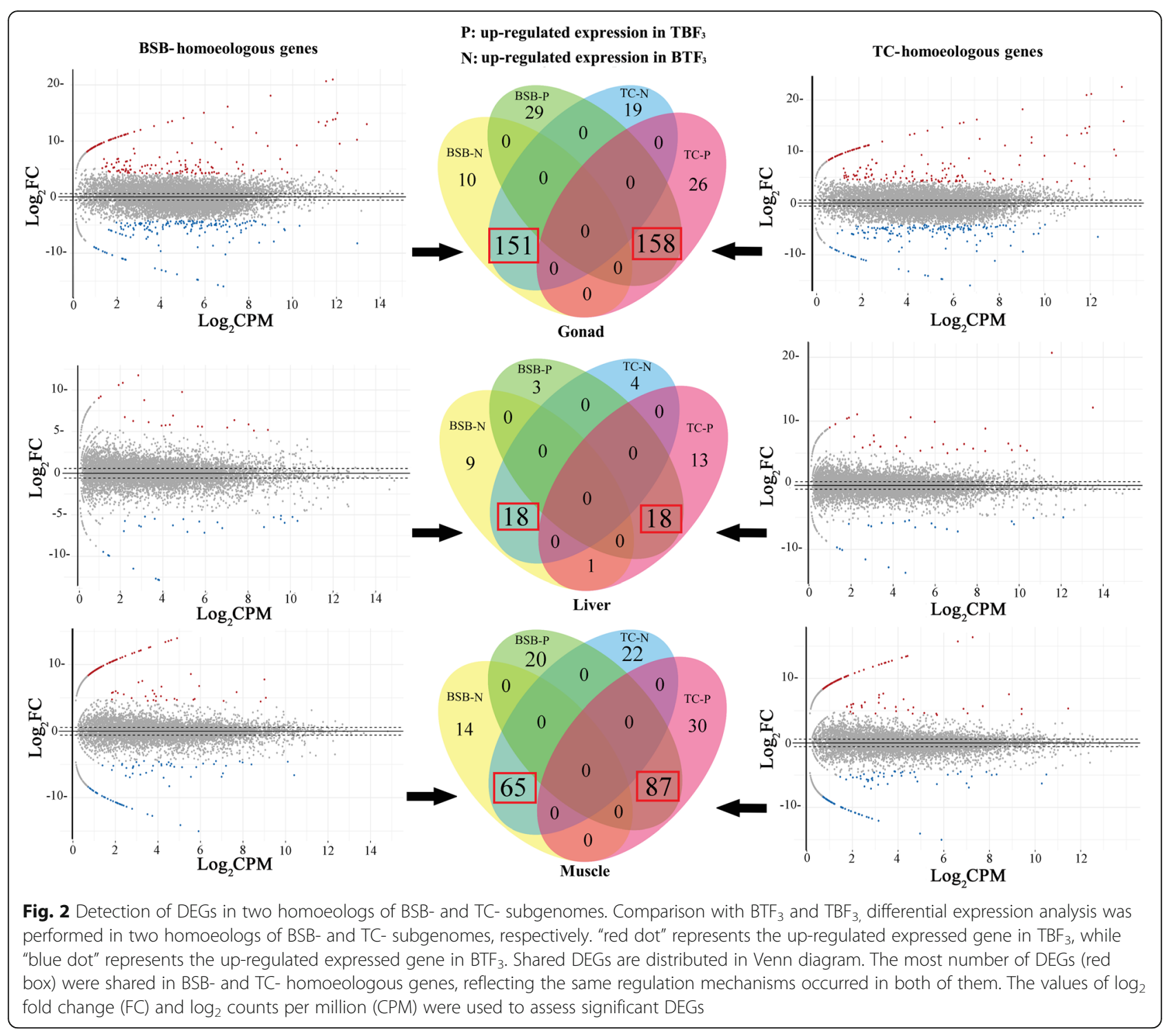

to homoeologous gene expression and AS events were found in reciprocal cross hybrids. However, the details of some AS events were inaccurate using Illumina data because of the short length of the reads. Therefore, long length reads of $\mathrm{BTF}_{3}$ PacBio data were used to improve the analysis of AS events in DEGs, including 38 AS events in gonad, 16 in muscle, and two in liver, while $\mathrm{TBF}_{3}$ data improved AS events in 33, 14, and six DEGs in gonad, muscle, and liver, respectively.

\section{AS distribution in bone morphology}

In view of the many shared traits between the reciprocal cross hybrids, the observed slight differences in their appearance made us consider their control of bone morphology regulated gene expression. Focusing on the BMP family, 17 orthologous genes were obtained from BSB and TC genomes, which exhibited gene expansion events (Fig. 3a). However, BSB- and TC- homoeologous gene expression in the three tissues studied was only detected in bmpr $2 a$ simultaneously. Slight differences in homoeologous gene expression were observed between $\mathrm{TBF}_{3}$ and $\mathrm{BTF}_{3}$ (Fig. 3b), although these were not significant. Therefore, bmpr $2 a$ was selected as a model to investigate AS events in BSB- and TC- homoeologs. We identified 12 exons, which was identical to the zebrafish (Fig. 3c) [23]. SE differences of two, one, and zero were detected between $\mathrm{TBF}_{3}$ and $\mathrm{BTF}_{3}$ in gonad, muscle, and liver, respectively, using Illumina data, but fewer AS events were detected in PacBio data. However, longer length transcripts provided more accurate AS predictions in respective BSB- and TC- homoeologs. In TChomoeologs of bmpr $2 a$, two RI events were observed between exons 9 and 10 and between exons 3 and 4 in $\mathrm{TBF}_{3}$, while three A3SS events and one SE were detected 


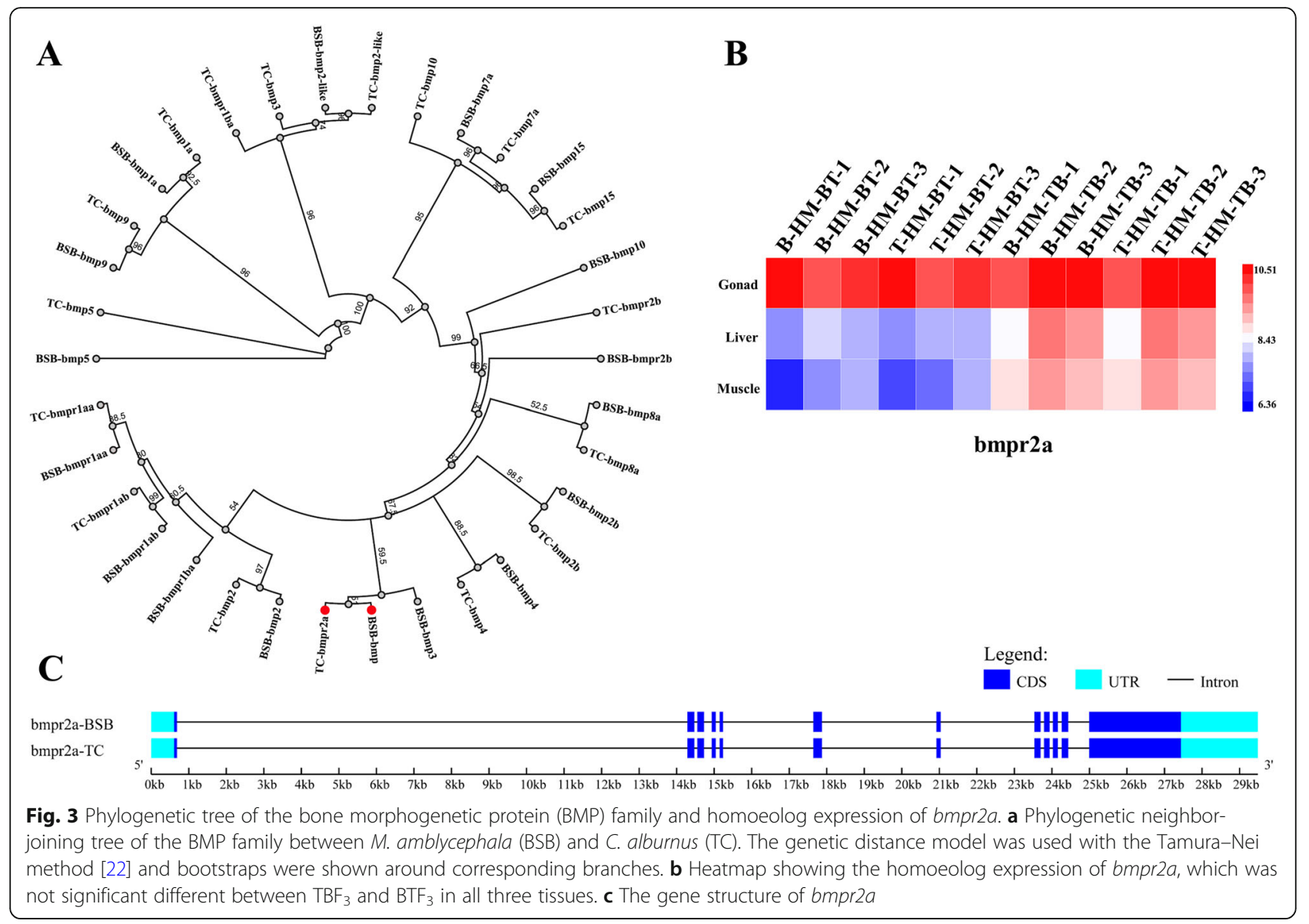

(Fig. 4). Unfortunately, we only detected one isoform because of the potential sequencing bias. In BSBhomoeologs of bmpr $2 a$, one SE event was identified that led to the loss of sequences in exons 7-11 of $\mathrm{TBF}_{3}$. Interestingly, we also identified an $\mathrm{SE}$ event with the loss of sequences in exons 2-11 in $\mathrm{BTF}_{3}$, similar to the $\mathrm{SE}$ event in the TC-homoeologous gene of $\mathrm{TBF}_{3}$ (Fig. 4).

For further determination of AS events in bmpr $2 a$, the transcripts in the muscle of $\mathrm{TB}$ and $\mathrm{BT}$ were sequenced by Sanger method. The one RI event between exons 9 and 10 (AS_2) and the two SE events distributed in parts of exon 12 (AS_3 and 4) were detected in TChomoeologs of $\mathrm{BTF}_{3}$ and $\mathrm{TBF}_{3}$. These phenomena were same to the above results of PacBio data (Fig. 4; Additional file 10: Fig. S6). Furthermore, one SE in exon 7 (AS_1) was observed in BSB-homoeologs of $\mathrm{BTF}_{3}$ and $\mathrm{TBF}_{3}$ (Additional file 10: Fig. S6).

\section{Discussion}

Hybrid, especially intergeneric hybrid, is a useful model to investigate homoeologs because of the more specific loci and SNPs that differ between the two subgenomes. Although plants have a large number of allodiploids and allopolyploids, the rarity in lower vertebrate species hinders our study of their expression. The establishment of two reciprocal cross hybrids of Megalobrama amblycephala and Culter alburnus $(2 \mathrm{n}=48)$, with the same chromosome numbers as their inbred parents, provides a useful model to study maternal effects, especially to regulation of mitochondrial DNA. It enabled us to obtain 20,131 species-specific orthologous gene pairs between M. amblycephala and C. alburnus.

Maternal effects may arise through mitochondrial DNA, cytoplasmic factors in the transmission of organelles, maternal environmental effects and so on [24, 25]. Our study only focused on the regulation of mitochondrial DNA. In the two reciprocal cross hybrids, mitochondria of the two species could lead to the different regulation pattern on energy metabolism by mitochondrial gene expression, and further change the growth characteristic, including body shape traits [26]. The slight differences in bone morphology between $\mathrm{BTF}_{3}$ and $\mathrm{TBF}_{3}$ provided us with an insight into the potential regulation of maternal effects. This represents an important field of study in evolutionary ecology, and there is an ongoing debate regarding their adaptive significance which acts to increase offspring fitness [27]. Here, we captured the expression diversity under the maternal 


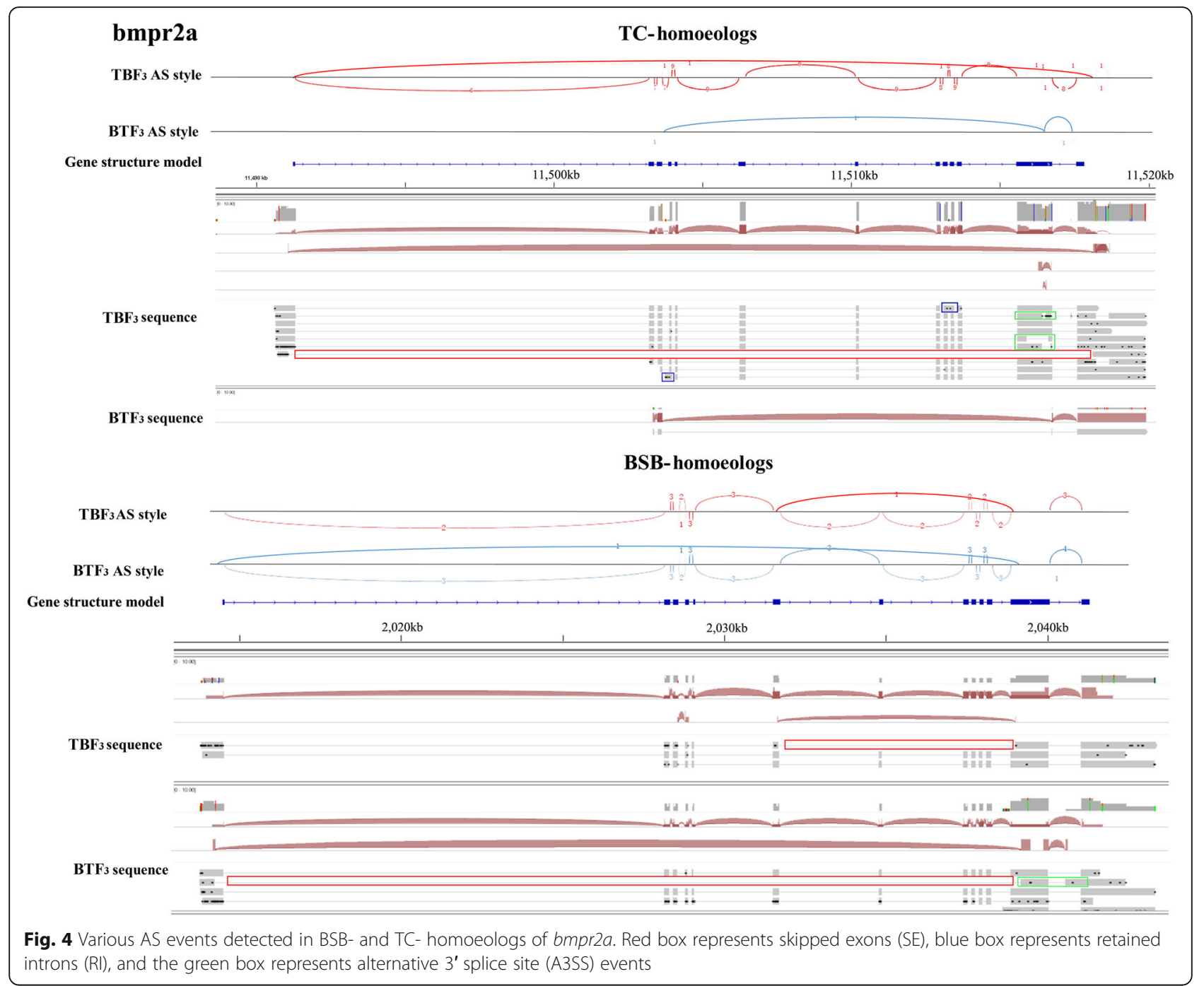

effects of M. amblycephala and C. alburnus. In a comparison of the two reciprocal cross hybrids, TChomoeologous genes exhibited slightly more differential expression than BSB-homoeologous genes (Fig. 2). This indicated that the maternal effect shaped the expression of both homoeologous genes, although there were few differences in DEGs between BSB- and TC- homoeologs. Furthermore, these results also showed that the maternal effects exhibited the different magnitudes in liver, muscle, and gonad. GO analysis of DEGs revealed that maternal effects could shape growth and immune functions by regulating corresponding gene expression.

AS is one of the most important components of genome functional complexity [28], and the resulting multiple transcripts lead to an abundance of gene expression profiles [8]. We identified 2402, 2959, and 3418 AS events between the two reciprocal cross hybrids in muscle, liver, and gonad, respectively, and PacBio sequencing resulted in a more accurate AS prediction, obtaining 76,518 isoforms in $\mathrm{TBF}_{3}$ and 82,083 in $\mathrm{BTF}_{3}$. The difference on AS number in each or orthologous gene pairs reflected the maternal effects contributed to AS changes (Fig. 1). These AS differences under maternal effects suggested various potential mechanisms. The analysis of human embryoid bodies revealed that the expression of histone deacetylase was regulated by maternal effects [29], while distinctive histone modification caused splice site switching by influencing the recruitment of splicing regulators via a chromatin-binding protein $[10,30]$. Furthermore, DNA methylation regulated the AS of mRNA precursors through two different mechanisms, including the elongation of RNA polymerase II by CCCTC-binding factor and methyl-CpG binding protein 2 [31]. On the other hand, expression divergence of homoeologs led to various expression patterns, including homoeolog expression bias and expression level dominance), further contributing to the formation of various phenotypes, including heterosis 
[17-19]. However, identification of homoeolog expression patterns with the corresponding phenotypes are challenging because of the complex gene regulatory network and unknown interactions between RNAs and proteins [32]. Studies on AS events on different homoeologs could help us gradually understanding of complex interactions among regulatory networks, in which AS events exhibits a close relationship with cis-regulation divergence [33]

Focusing on AS and expression in the BMP family in our study, we investigated the relationship of gene diversity and phenotypic changes in body shape between two reciprocal cross hybrids (Figs. 3 and 4). BMPs are involved in a host of cellular functions including osteogenesis, cell growth, and cell differentiation [34]. Among the family members, bmpr $2 a$ encodes a transmembrane serine/threonine kinase that plays an important role in follicle development in preparation for ovulation [35]. The ratio of multiple alternative spliced variants of isoform-A (full-length) to isoform-B (missing exon 12) regulates heritable pulmonary arterial hypertension in humans [36], although it may not be the main effect in the morphological difference. Except full-length isoform in $b m p r 2 a$, we further detected seven other isoforms in the TC- homoeologous gene, but only one in the BSBhomoeologous gene because of the low sequencing coverage. Therefore, additional PacBio sequencing data of hybrids and their inbred parents are required for future studies. Nevertheless, our results provide evidence of how maternal effects shape expression diversity.

\section{Conclusions}

In this work, we focused on the changes of mRNA expression and AS related to maternal effects in the two reciprocal cross hybrids. First, the 49-348 differentially expressed BSB-homoeologous genes and 54-354 differentially expressed TC-homoeologous genes were detected between the two reciprocal cross hybrids. Second, 2402, 2959, and 3418 AS events were detected in three tissues (muscle, liver, and gonad) between the two reciprocal cross hybrids, respectively. PacBio data further improved AS prediction of Illumina data. Third, we detected various AS patterns in bmpr2a, which exhibited their potential effects to body shape between the two reciprocal cross hybrids. These findings provide a novel insight into mRNA expression changes and AS under maternal effects in lower vertebrates.

\section{Methods}

\section{Animal materials}

Fertile reciprocal cross hybrids $(2 \mathrm{n}=48)$ were obtained from hybridization between $M$. amblycephala $\times C$. alburnus, and $F_{2}$ and $F_{3}$ generation offspring were obtained from the self-crossing of $F_{2}$ and $F_{3}$ reciprocal cross offspring $[20,21]$. The allodiploid lineage then continued into $\mathrm{F}_{5}$ by successive self-crossing. Among these hybrid offspring, only chimeric offspring with half of the two inbred parental genomes (1:1) identified with 45S rDNA were used in our study [21]. These fishes were cultured to 2 years of age under the same suitable environment, including water temperature, dissolved oxygen content and adequate forage at the Engineering Center of Polyploidy Fish Breeding of the National Education Ministry, Hunan Normal University, Hunan, China. After collecting, the six reciprocal cross hybrids of $F_{3}$ (three individuals in $\mathrm{BT}$ and three individuals in $\mathrm{TB})$ were kept in the same aquarium $\left(23^{\circ} \mathrm{C}\right)$ for 2 days. Then, these fishes were deeply anaesthetized with 100 $\mathrm{mg} / \mathrm{L}$ MS-222 (Tricaine Methanesulfonate) (Sigma-Aldrich, St Louis, MO, USA) for $10 \mathrm{~min}\left(25^{\circ} \mathrm{C}\right)$ in a separate tank. Then, the more powder of MS-222 was added and mixed with water in the tank for increasing concentration to $300 \mathrm{mg} / \mathrm{L}$. After checking the death of the fishes, they was moved from water for dissection. All tissue samples were excised carefully and subsequently stored at $-80^{\circ} \mathrm{C}$. These experiments were conducted at the Engineering Center of Polyploidy Fish Breeding of the National Education Ministry, Hunan Normal University, Hunan, China, and conformed to the National Institutes of Health Guide for Care and Use of Laboratory Animals. The animal work was approved by the academic committee in Hunan Normal University (approval ID: 01/2018).

Illumina reads of $\mathrm{BTF}_{3}, \mathrm{TBF}_{3}$, and their inbred parents (M. amblycephala and C. alburnus) as well as liver, muscle, and gonad tissues were downloaded from the NCBI Sequence Read Archive database (accession numbers: SRS404403, SRS4069048, SRS47368, and SRS805925). Raw reads containing adapters or ploy-N and low-quality reads were removed using in-house perl scripts. High-quality reads were used in subsequent analysis.

\section{Characteristics of two inbred parental genomes}

Assembled genome of the two inbred parents (BSB and TC) help understand their gene structures (accession number: PRJNA269572 in NCBI BioProject database). Although lacking the genomes of hybrid offspring, we used the combined genomes representing the in silico genomes of hybrids. Exon numbers and the number and length of coding sequences (CDS) of the two genomes were annotated with Kyoto Encyclopedia of Genes and Genomes (KEGG) and Gene Ontology (GO) databases. Orthologous gene pairs between BSB and TC were determined by the best reciprocal BLAST hits with each other with an e-value of $1 \mathrm{e}^{-5}$. The Ks value between the orthologous pairs was calculated by the yn 00 program in PAML package. Then, the 17 orthologous gene pairs of 
bone morphogenetic protein (BMP) family were obtained based on the previous annotation results.

\section{Mapping of RNA sequencing (RNA-seq) data}

All Illumina reads of $M$. amblycephala and C. alburnus were aligned to $M$. amblycephala and C. alburnus genomes using Star (v 2.4.0) with default parameters [37]. mRNA-seq reads of $\mathrm{TB}$ and $\mathrm{BTF}_{3}$ were aligned to the combined nuclear and mitochondrial genomes of $M$. amblycephala (accession number: MF522177.1) and $C$. alburnus (accession number: KX244762.1). The numbers of mapping counts in each gene were calculated with inhouse perl scripts. To avoid the negative effect of expression noise, expression analysis was only performed on filtered genes with mapping read counts $\geq 5$ in all three biological replicates in each contrast. The total expression value was normalized to the ratio of the number of mapped reads at each gene to the total number of mapped reads for the entire genome. Orthologous genes between $M$. amblycephala and C. alburnus were obtained from all-against-all reciprocal BLASTP (v 2.2.26) comparisons with the parameters of "-e 10-5 -F -v 1 $\mathrm{m}$ 8" based on protein sequences. Transcripts lacking annotated CDSs and those of length $<100 \mathrm{bp}$ were discarded.

\section{Detection of homoeologous gene expression in reciprocal cross hybrids}

To investigate the expression level of parent-of-origin genes (homoeologous gene expression), the LASTZ pairwise alignment tool ( $v$ 1.02.00) [38] with default parameters was used to obtain corresponding loci from the orthologous gene pairs between $M$. amblycephala and $C$. alburnus. InDel loci were discarded, and only aligned loci with the best scoring match were used for the next analysis. Single nucleotide polymorphisms (SNPs) were collected using the SNP Calling pipeline with GATK (v 3.8) based on the results of parent transcriptome mapping to respective genomes. According to the comparison of SNPs and other loci in orthologous gene pairs between $M$. amblycephala and $C$. alburnus, loci with complete differences including heterozygous and homozygous loci were considered to be species-specific SNPs as seen in Schaefke et al $[39,40]$. To remove the negative effect of sequencing and mapping, the screening of species-specific SNPs was checked for consistency in three biological replicates, and loci possessing read counts $\geq 1$ in all accessions and biological replicates in each contrast were retained.

To describe homoeologous gene expression in hybrids, hybrid mapping results (bam files) of transcriptomes were used in our next analysis. The mapping files of each hybrid were divided into two categories based on parent reference genomes. $\mathrm{BSB}-/ \mathrm{TC}$ - homoeolog reads in the hybrid had been calculated using in-house perl scripts, based on corresponding BSB-/TC- speciesspecific SNPs in their corresponding mapping files. The expression levels of parents in homoeolog analysis were also counted based on respective reference genomes related to species-specific SNPs. To remove the negative effect of hybrid mutation sites in biological replicates, abnormal values were discarded when estimating BSBand TC- homoeolog expression levels if the mapping reads of the species-specific SNP did not comply with the threshold of mean $\pm 2 \mathrm{SD}$ in three biological replicates. Then, the sum counts of BSB- and TC- homoeologs in each gene were normalized based on the ratio of the number of mapped reads at each gene to the total number of mapped reads for the entire genome [41]. The sum of BSB-/TC- homoeolog reads in all speciesspecific SNPs of each gene was used to assess BSB-/TChomoeolog expression. Differentially expressed genes (DEGs) were analyzed with the edgeR package of the $R$ program (version 2.13) ( $\mathrm{R}$ Foundation for Statistical Computing, Vienna, Austria). A false discovery rate threshold $\leq 0.05$ was used in the analysis.

\section{AS analysis of Illumina data}

After classifying the two homoeologs (BSB and TC), we further analyzed AS events in $\mathrm{TBF}_{3}$ and $\mathrm{BTF}_{3}$ using Illumina data. Mapped reads were assembled to transcripts using Cufflinks software (v2.2.1). Transcripts from three biological replicates were then merged together. AS events in two respective homoeologs were predicted using ASprofile [42], and five AS events (mutually exclusive exons [MXE], SE, RI, A5SS, and A3SS) were detected in the above analysis pipeline.

\section{RNA isolation, library construction, and PacBio sequencing}

To sequence the transcriptomes of reciprocal cross hybrids and their inbred parents, total RNA was isolated and purified by TRIzol LS reagent (Invitrogen Corp.,). The RNA concentration was measured using a Nanodrop spectrophotometer. Total RNA samples were treated with DNase I (Invitrogen) to remove any contaminating genomic DNA. The purified RNA was quantified using a 2100 Bioanalyzer system (Agilent, Santa Clara, CA, USA).

To analyze AS in reciprocal cross $F_{3}$ hybrids, nonassembled long-length reads underwent PacBio sequencing. Total RNA of $\mathrm{TBF}_{3}$ and $\mathrm{BTF}_{3}$ from liver, muscle, and gonad was mixed in equal amounts. It was then reverse-transcribed using the SMARTer PCR CDNA Synthesis Kit and PCR-amplified using KAPA HiFi PCR kits (Kapa Biosystems). PCR products $0.5-6 \mathrm{~kb}$ and $>6$ $\mathrm{kb}$ were selected using agarose gel electrophoresis. Libraries were then constructed from these cDNA 
products using the SMRTbell Template Prep Kit 1.0. After library preparation, the library template and enzyme mixture were used for sequencing in the PacBio Sequel $^{\text {Tx }}$ system.

\section{AS analysis of PacBio data}

After obtaining the sequencing data, adaptors were deleted using SMRTLINK (v 5.0.1) [43, 44]. Low-quality data (such as adaptor sequences, subreads $<50 \mathrm{bp}$, and reads with accuracy rates $<0.75$ ) were deleted from the raw data. Sequence reads from the PacBio RS SMRT chip were processed using the PacBio SMRT-Portal analysis suite to generate circular consensus sequences (CCSs). To obtain greater accuracy, the reads (CCS cycles $>1$ and accuracy $>0.8)$ were used to obtain fulllength reads ( $>300 \mathrm{bp}$, poly (A) tails, $5^{\prime}$ primers, and $3^{\prime}$ primers) based on the SMRT Iso-Seq analysis pipeline (http://www.pacb.com/products-and-services/analyticalsoftware/smrt-analysis/). Full and reduced length transcripts were determined with both the $\mathrm{m}^{7} \mathrm{G}$-cap structure and the poly(A) tail. Then, redundant sequences were clustered using the ICE algorithm, and consensus sequences were obtained by pbdagcon (https://github. com/PacificBiosciences/pbdagcon) with a Directed Acyclic Graph Consensus algorithm. Illumina reads of reciprocal cross hybrids were used to rectify sequencing errors using Lordec [45].

The above data were aligned to the combined reference genome sequences of $M$. amblycephala and $C$. alburnus using default parameters of GMAP software [46]. A custom Python script (alternative_splice.py, https://github.com/Nextomics/pipeline-for-isoseq) was used to identify AS events from alignments which were classified into MXE, SE, RI, A5SS, and A3SS. To obtain homoelogue AS in $\mathrm{BT}$ and $\mathrm{TBF}_{3}$, we focused on orthologous gene pairs between M. amblycephala and C. alburnus. Species-specific SNPs obtained from mRNA-seq data were used to rectify the mapping results of PacBio sequencing data. The threshold of parental homoelogue sequences was $85 \%$ of species-specific SNPs. Under this condition, the expression of BSB-/TC- homoeologs in $\mathrm{BT}$ and $\mathrm{TBF}_{3}$ was exhibited by the Integrative Genomics Viewer [47].

\section{AS determination in bmpr2a using sanger data}

For checking the AS events in bmpr $2 a$, total RNA extracted from muscle of $\mathrm{BTF}_{3}$ and $\mathrm{TBF}_{3}$ was treated with gDNA Eraser (TaKaRa). Then, first-strand cDNA was synthesized using AMV reverse transcriptase (Fermentas, Canada Inc.) with an oligo $(\mathrm{dT})_{12-18}$ primer. The primers (5'- TTGAAGGCCGAATAACAATTCTT-3', 3'-AGTCATTAGGATCTGAGAAGCGAG-5') used to amplify the cDNA fragment of $b m p r 2 a$ by PCR. The length of transcripts were exhibited by agarose gel electrophoresis. PCR-amplified products were separated on a $1.2 \%$ agarose gel using TBE buffer. The targeted fragments were purified using a gel extraction kit (Sangon,Shanghai, China) and ligated into the pMD18-T vector (TaKaRa, Dalian, China). The plasmids were transformed into E.coli DH5 $\alpha$ and purified. The inserted targeted fragments in the pMD18-T vector were sequenced using Sanger sequencing. AS events were analyzed with BioEdit [48] and Clustal W [49].

\section{Supplementary information}

Supplementary information accompanies this paper at https://doi.org/10. 1186/s12864-020-06866-7.

Additional file 1: Table S1. The summary of 45S rDNA in the two reciprocal cross hybrids.

Additional file 2: Table S2. The determination of maternal expression of mitochondrial gene in the two reciprocal cross hybrids.

Additional file 3: Figure S1. Distribution of gene lengths and exon numbers.

Additional file 4: Figure S2. Shared genes between the two reciprocal cross hybrids detected by PacBio sequencing.

Additional file 5: Table S3. Differential expression between the two reciprocal cross hybrids detected in gonad, liver and muscle.

Additional file 6: Figure S3. Gene ontology (GO) categories (level 2) of DEGs in gonad, liver, and muscle.

Additional file 7: Figure S4. The distribution of DEGs in the biological process category (level 3) of gene ontology (GO).

Additional file 8: Table S4. The AS events and their gene distribution predicted from Illumina data.

Additional file 9: Figure S5. Distribution of genes with the high AS events (AS number $\geq 5$ ) in gonad, liver, and muscle.

Additional file 10: Figure S6. AS events of bmpr2a detected by Sanger sequencing. (A) The model of AS events in $\mathrm{TBF}_{3}$ and $B \mathrm{BF}_{3} . \mathrm{TC}$ and $\mathrm{BSB}$ represent the complete coding sequences in parental TC and BSB, respectively. AS_1 is the SE event in BSB-homoeologs, while AS_2, 3 and 4 are the AS events in TC-homoeologs. (B) The sequence alignments of the complete coding sequences in $\mathrm{TC}$ and $\mathrm{BSB}$ and the four $\mathrm{AS}$ in $\mathrm{TBF}_{3}$ and $\mathrm{BTF}_{3}$.

\section{Abbreviations}

CCSs: Circular consensus sequences; MXE: Mutually exclusive exons; DEGs: Differentially expressed genes; SNPs: Single nucleotide polymorphisms; RNA-seq: RNA sequencing; KEGG: Kyoto Encyclopedia of Genes and Genomes; GO: Gene Ontology; CDS: Coding sequences; BSB: Megalobrama amblycephala; TC: Culter alburnus; BT and TB: Two reciprocal cross hybrids of M. amblycephala and C. alburnus; AS: Alternative splicing; SE: Skipped exons; RI: Retained introns; A5SS: Alternative 5' splice sites; A3SS: Alternative 3' splice sites; AP: Alternative position

\section{Acknowledgements}

We thank Sarah Williams, PhD, from Liwen Bianji, Edanz Group China (www. liwenbianji.cn), for editing the English text of a draft of this manuscript.

\section{Authors' contributions}

LR wrote the manuscript. SJL modified the manuscript. $L R$ and $S J L$ contributed to the conception and design of the study. LR, XG, PCY, and JLC carried out bioinformatics analyses. XJY, WHL, CW, and CCT provided assistance in extracting the raw material. The authors read and approved the final manuscript.

\section{Funding}

This research was supported by the National Natural Science Foundation of China (Grant Nos. 31730098, 31430088, and U19A2040), the Key Research 
and Development Program of Hunan Province (Grant No. 2018NK2072), the earmarked fund for China Agriculture Research System (Grant No. CARS-45), the Key Research and Development Program of Hunan Province (Grant No. 2018NK2072), the Hunan Provincial Natural Science and Technology Major Project (Grant No. 2017NK1031), and the Cooperative Innovation Center of Engineering and New Products for Developmental Biology of Hunan Province (Grant No. 20134486). High-level Talent Agglomeration Program of Hunan, China (2019RS1044) to Shaojun Liu; the National Natural Science Foundation of China (Grant Nos. 31702334), the Key Research and Development Project of Hunan Province (Grant No. 2016NK2128) to Li Ren.

\section{Availability of data and materials}

Raw data of PacBio sequencing have been submitted to the NCBI sequence read archive under accession numbers SRX5043671 and SRX5043670. Illumina reads of $\mathrm{BTF}_{3}, \mathrm{TBF}_{3}$, and their inbred parents (M. amblycephala and C. alburnus) as well as liver, muscle, and gonad tissues were downloaded from the NCBI Sequence Read Archive database (accession numbers: SRS404403, SRS4069048, SRS47368, and SRS805925). Assembled genomes of M. amblycephala and C. alburnus were downloaded from NCBI BioProject database (accession number: PRJNA269572). The mitochondrial genomes of M. amblycephala and C. alburnus were downloaded from accession numbers of MF522177.1 and KX244762.1 in NCBI Nucleotide Database, respectively.

\section{Ethics approval and consent to participate}

The animal work was approved by the academic committee in Hunan Normal University (approval ID: 01/2018). We declare that animal handling complied with the relevant guidelines of the National Institutes of Health Guide for Care and Use of Laboratory Animals and the Engineering Center of Polyploidy Fish Breeding of the National Education Ministry, Hunan Normal University, Hunan, China.

\section{Consent for publication}

Not Applicable.

\section{Competing interests}

The authors declare that they have no competing interests.

\section{Author details}

${ }^{1}$ State Key Laboratory of Developmental Biology of Freshwater Fish, Hunan Normal University, Changsha 410081, Hunan, P.R. China. ${ }^{2}$ College of Life Sciences, Hunan Normal University, Changsha 410081, Hunan, P.R. China. ${ }^{3}$ Tang Tang Biomedical Technology (BeiJing) Co., Ltd., Beijing, P.R. China.

\section{Received: 27 May 2019 Accepted: 23 June 2020}

\section{Published online: 02 July 2020}

\section{References}

1. Wolf JB, Wade MJ. What are maternal effects (and what are they not)? Philosophical Transact Royal Soc B: Biol Sci. 2009;364(1520):1107-15.

2. Green BS. Maternal effects in fish populations. Adv Mar Biol. 2008;54:1-105.

3. Berkeley SA, Chapman C, Sogard SM. Maternal age as a determinant of larval growth and survival in a marine fish, Sebastes melanops. Ecology. 2004;85(5):1258-64.

4. Howell CY, Bestor TH, Ding F, Latham KE, Mertineit C, Trasler JM, et al. Genomic imprinting disrupted by a maternal effect mutation in the Dnmt1 gene. Cell. 2001;104(6):829-38.

5. Bourc'his D, Xu G-L, Lin C-S, Bollman B, Bestor TH. Dnmt3L and the establishment of maternal genomic imprints. Science. 2001;294(5551): 2536-9.

6. Bartolomei MS, Tilghman SM. Genomic imprinting in mammals. Annu Rev Genet. 1997;31(1):493-525.

7. Reik W, Dean W, Walter J. Epigenetic reprogramming in mammalian development. Science. 2001;293(5532):1089-93.

8. Nilsen TW, Graveley BR. Expansion of the eukaryotic proteome by alternative splicing. Nature. 2010;463(7280):457.

9. Luco RF, Allo M, Schor IE, Kornblihtt AR, Misteli T. Epigenetics in alternative pre-mRNA splicing. Cell. 2011;144(1):16-26.

10. Luco RF, Pan Q, Tominaga K, Blencowe BJ, Pereira-Smith OM, Misteli T. Regulation of alternative splicing by histone modifications. Science. 2010; 327(5968):996-1000.
11. Pan Q, Shai O, Lee LJ, Frey BJ, Blencowe BJ. Deep surveying of alternative splicing complexity in the human transcriptome by high-throughput sequencing. Nat Genet. 2008;40(12):1413.

12. Gerstein MB, Lu ZJ, Van Nostrand EL, Cheng C, Arshinoff BI, Liu T, et al. Integrative analysis of the Caenorhabditis elegans genome by the modENCODE project. Science. 2010;330(6012):1775-87.

13. Graveley BR, Brooks AN, Carlson JW, Duff MO, Landolin JM, Yang L, et al. The developmental transcriptome of Drosophila melanogaster. Nature. 2011;471(7339):473.

14. Necsulea A, Kaessmann H. Evolutionary dynamics of coding and noncoding transcriptomes. Nat Rev Genet. 2014;15(11):734.

15. Lappalainen T, Sammeth M, Friedländer MR, AC't Hoen P, Monlong J, Rivas $M A$, et al. Transcriptome and genome sequencing uncovers functional variation in humans. Nature. 2013;501(7468):506.

16. Mallet J. Hybrid speciation. Nature. 2007:446(7133):279-83.

17. Yoo MJ, Szadkowski E, Wendel JF. Homoeolog expression bias and expression level dominance in allopolyploid cotton. Heredity. 2013;110(2): $171-80$.

18. Grover CE, Gallagher JP, Szadkowski EP, Yoo MJ, Flagel LE, Wendel JF. Homoeolog expression bias and expression level dominance in allopolyploids. New Phytol. 2012;196(4):966-71.

19. Li A, Liu D, Wu J, Zhao X, Hao M, Geng S, et al. mRNA and small RNA transcriptomes reveal insights into dynamic Homoeolog regulation of allopolyploid Heterosis in nascent Hexaploid wheat. Plant Cell. 2014;26(5): 1878-900.

20. Xiao J, Kang X, Xie L, Qin Q, He Z, Hu F, et al. The fertility of the hybrid lineage derived from female Megalobrama amblycephala $x$ male Culter alburnus. Anim Reprod Sci. 2014;151(1-2):61-70.

21. Xiao J, Hu F, Luo K, Li W, Liu S. Unique nucleolar dominance patterns in distant hybrid lineage derived from Megalobrama amblycephala $\times$ Culter alburnus. BMC Genet. 2016;17(1).

22. Tamura K, Nei M. Estimation of the number of nucleotide substitutions in the control region of mitochondrial DNA in humans and chimpanzees. Mol Biol Evol. 1993;10(3):512-26.

23. Monteiro R, van Dinther M, Bakkers J, Wilkinson R, Patient R, ten Dijke P, et al. Two novel type II receptors mediate BMP signalling and are required to establish left-right asymmetry in zebrafish. Dev Biol. 2008;315(1):55-71.

24. Wulff RD. Environmental maternal effects on seed quality and germination. In: Seed development and germination. New York: Routledge; 2017. p. 491505.

25. Ainsworth HF, Unwin J, Jamison DL, Cordell HJ. Investigation of maternal effects, maternal-fetal interactions and parent-of-origin effects (imprinting), using mothers and their offspring. Genet Epidemiol. 2011;35(1):19-45.

26. Spiegelman BM, Flier JS. Obesity and the regulation of energy balance. Cell. 2001;104(4):531-43.

27. J. Marshall D, Uller T. When is a maternal effect adaptive? Oikos. 2007; 116(12):1957-63.

28. Modrek B, Lee C. A genomic view of alternative splicing. Nat Genet. 2002; 30(1):13-9.

29. Sun C, Denisenko O, Sheth B, Cox A, Lucas ES, Smyth NR, et al. Epigenetic regulation of histone modifications and Gata6 gene expression induced by maternal diet in mouse embryoid bodies in a model of developmental programming. BMC Dev Biol. 2015;15:3.

30. Zhou HL, Luo G, Wise JA, Lou H. Regulation of alternative splicing by local histone modifications: potential roles for RNA-guided mechanisms. Nucleic Acids Res. 2014;42(2):701-13.

31. Lev Maor G, Yearim A, Ast G. The alternative role of DNA methylation in splicing regulation. Trends Genet. 2015;31(5):274-80.

32. Emerson JJ, Hsieh LC, Sung HM, Wang TY, Huang CJ, Lu HH, et al. Natural selection on cis and trans regulation in yeasts. Genome Res. 2010;20(6):826-36.

33. Gao Q, Sun W, Ballegeer M, Libert C, Chen W. Predominant contribution of cis-regulatory divergence in the evolution of mouse alternative splicing. Mol Syst Biol. 2015;11(7):816.

34. Furuta Y, Piston DW, Hogan BL. Bone morphogenetic proteins (BMPs) as regulators of dorsal forebrain development. Development. 1997;124(11):2203-12.

35. Juengel JL, Hudson NL, Heath DA, Smith P, Reader KL, et al. Growth differentiation factor 9 and bone morphogenetic protein 15 are essential for ovarian follicular development in sheep. Biol Reprod. 2002;67(6):1777-89.

36. Cogan J, Austin E, Hedges L, Womack B, West J, Loyd J, et al. Role of BMPR2 alternative splicing in heritable pulmonary arterial hypertension penetrance. Circulation. 2012;126(15):1907-16. 
37. Bennett BL, Sasaki DT, Murray BW, O'Leary EC, Sakata ST, Xu W, et al. SP600125, an anthrapyrazolone inhibitor of Jun N-terminal kinase. Proc Natl Acad Sci U S A. 2001;98(24):13681-6.

38. Harris RS. Improved pairwise alignment of genomic DNA: PhD thesis. University Park: Pennsylvania State University; 2007.

39. Schaefke B, Emerson JJ, Wang TY, Lu MY, Hsieh LC, Li WH. Inheritance of gene expression level and selective constraints on trans- and cis-regulatory changes in yeast. Mol Biol Evol. 2013;30(9):2121-33.

40. McManus CJ, Coolon JD, Duff MO, Eipper-Mains J, Graveley BR, Wittkopp PJ. Regulatory divergence in drosophila revealed by mRNA-seq. Genome Res. 2010;20(6):816-25.

41. Quackenbush J. Microarray data normalization and transformation. Nat Genet. 2002;32:496-501.

42. Florea L, Song L, Salzberg SL. Thousands of exon skipping events differentiate among splicing patterns in sixteen human tissues. F1000Research. 2013;2:188.

43. Ardui S, Ameur A, Vermeesch JR, Hestand MS. Single molecule real-time (SMRT) sequencing comes of age: applications and utilities for medical diagnostics. Nucleic Acids Res. 2018;46(5):2159-68.

44. Reiner J, Pisani L, Qiao W, Singh R, Yang Y, Shi L, et al. Cytogenomic identification and long-read single molecule real-time (SMRT) sequencing of a Bardet-Biedl syndrome 9 (BBS9) deletion. NPJ Genom Med. 2018;3:3.

45. Salmela L, Rivals E. LoRDEC: accurate and efficient long read error correction. Bioinformatics. 2014:30(24):3506-14.

46. Wu TD, Watanabe CK. GMAP: a genomic mapping and alignment program for mRNA and EST sequences. Bioinformatics. 2005;21(9):1859-75.

47. Thorvaldsdottir H, Robinson JT, Mesirov JP. Integrative genomics viewer (IGV): high-performance genomics data visualization and exploration. Brief Bioinform. 2013;14(2):178-92.

48. Hall TA. BioEdit: a user-friendly biological sequence alignment editor and analysis program for windows 95/98/NT. Nucleic Acids Symp Ser. 1999;41: 95-8.

49. Larkin MA, Blackshields G, Brown NP, Chenna R, McGettigan PA, McWilliam $H$, et al. Clustal W and Clustal X version 2.0. Bioinformatics. 2007;23(21): 2947-8.

\section{Publisher's Note}

Springer Nature remains neutral with regard to jurisdictional claims in published maps and institutional affiliations.

Ready to submit your research? Choose BMC and benefit from:

- fast, convenient online submission

- thorough peer review by experienced researchers in your field

- rapid publication on acceptance

- support for research data, including large and complex data types

- gold Open Access which fosters wider collaboration and increased citations

- maximum visibility for your research: over $100 \mathrm{M}$ website views per year

At BMC, research is always in progress.

Learn more biomedcentral.com/submissions 